PROCEEDINGS OF THE

AMERICAN MATHEMATICAL SOCIETY

Volume 132, Number 3, Pages 691-695

S 0002-9939(03)07104-1

Article electronically published on July 16, 2003

\title{
$2 S_{4} * Q_{8}$-EXTENSIONS IN CHARACTERISTIC 3
}

\author{
TERESA CRESPO
}

(Communicated by Stephen D. Smith)

\begin{abstract}
We present an explicit construction of the complete family of Galois extensions of a field $K$ of characteristic 3 with Galois group the central product $2 S_{4} * Q_{8}$ of a double cover $2 S_{4}$ of the symmetric group $S_{4}$ and the quaternion group $Q_{8}$, containing a given $S_{4}$-extension of the field $K$.
\end{abstract}

Let $2 S_{4} * Q_{8}$ be the central product of a double cover $2 S_{4}$ of the symmetric group $S_{4}$ and the quaternion group $Q_{8}$. In [2, we gave a method of construction of $2 S_{4} * Q_{8}$-extensions of a field $K$ of characteristic different from 2 . In this paper we examine more closely the characteristic 3 case. Given an $S_{4}$-extension $L_{1}$ of a field $K$ containing the finite field $\mathbb{F}_{9}$ of 9 elements, we give explicitly all $2 S_{4} * Q_{8}$-extensions of $K$ containing $L_{1}$. In the case when $K=k\left(\left(Y_{1}, Y_{2}, Y_{3}\right)\right)$, the quotient field of the formal power series ring $R=k\left[\left[Y_{1}, Y_{2}, Y_{3}\right]\right]$ in 3 variables over an algebraically closed field $k$ of characteristic 3 , the determination of all $2 S_{4} * Q_{8}$-extensions of $K$ is interesting in the framework of Abhyankar's Normal Crossings Local Conjecture (see [1). As mentioned by Abhyankar in [1], the groups $2 S_{4} * Q_{8}$ are examples of groups $G$ such that the quotient $G / 3(G)$ of $G$ by the subgroup $3(G)$ generated by all of its 3-Sylow subgroups is abelian, generated by 3 generators, but $3(G)$ does not have an abelian supplement in $G$, i.e., an abelian subgroup of $G$ generating $G$ together with $3(G)$. By a result of Harbater et al. (see [4]), this property makes them the "simplest" groups not appearing as Galois groups of a Galois extension $L$ of $K$ such that $R Y_{1}, R Y_{2}, R Y_{3}$ are the only height-one primes in $R$ that are possibly ramified in $L$. We note that Harbater and Lefcourt (see [6]) have shown that every finite group can be obtained as a ramified extension of $k\left(\left(Y_{1}, Y_{2}, Y_{3}\right)\right)$. The results in this paper provide an explicit construction of $2 S_{4} * Q_{8}$-extensions in the characteristic 3 case. As a first step towards the determination of the ramification locus of the corresponding coverings, we give a formula for the discriminant.

Let us first recall the definitions and fix notation. We denote by $2 S_{n}$ one of the two double covers of the symmetric group $S_{n}$ reducing to the nontrivial double cover $2 A_{n}$ of the alternating group $A_{n}$ and by $Q_{8}$ the quaternion group, which is a double cover of the Klein group $V_{4}$. The group $2 S_{4} * Q_{8}$ is the central product of $2 S_{4}$ and $Q_{8}$. Let $L_{1} \mid K$ be a Galois extension with Galois group the symmetric group $S_{4}$, for $K$ a field of characteristic different from 2 . We assume that $L_{1}$ is given as the splitting field of a polynomial $P(X) \in K[X]$ of degree 4 . We want to determine when $L_{1}$ is embeddable in a Galois extension of $K$ with Galois group

Received by the editors July 18, 2002 and, in revised form, November 13, 2002.

2000 Mathematics Subject Classification. Primary 12F12.

Partially supported by the grant BFM2000-0794-C02-01 of the Spanish Ministry of Education.

(C)2003 American Mathematical Society 
$2 S_{4} * Q_{8}$. This fact is equivalent to the existence of a Galois extension $L_{2} \mid K$ with Galois group $V_{4}$, disjoint from $L_{1}$, and such that, if $L$ is the compositum of $L_{1}$ and $L_{2}$, the Galois embedding problem

$$
2 S_{4} * Q_{8} \rightarrow S_{4} \times V_{4} \simeq \operatorname{Gal}(L \mid K)
$$

is solvable. We recall that a solution to this embedding problem is a quadratic extension field $\widetilde{L}$ of the field $L$, which is a Galois extension of $K$ with Galois group $2 S_{4} * Q_{8}$ and such that the restriction epimorphism between the Galois groups $\operatorname{Gal}(\widetilde{L} \mid K) \rightarrow \operatorname{Gal}(L \mid K)$ agrees with the given epimorphism $2 S_{4} * Q_{8} \rightarrow S_{4} \times V_{4}$. If $\widetilde{L}=L(\sqrt{\gamma})$ is a solution, then the general solution is $L(\sqrt{r \gamma}), r \in K^{*}$. Given a Galois extension $L_{1} \mid K$ with Galois group $S_{4}$, in order to obtain all $2 S_{4} * Q_{8^{-}}$ extensions of $K$ containing $L_{1}$, we have to determine all $V_{4}$-extensions $L_{2}$ of $K$, disjoint from $L_{1}$, and such that the embedding problem (11) is solvable.

Let us now specify notation by writing $2^{+} S_{n}$ or $2^{-} S_{n}$ depending on whether transpositions in $S_{n}$ lift in the double cover to involutions or to elements of order 4. Let $E=K[X] /(P(X))$, for $P(X)$ the polynomial of degree 4 realizing $L_{1}$, let $Q_{E}$ denote the trace form of the extension $E \mid K$, i.e., $Q_{E}(x)=\operatorname{Tr}_{E \mid K}\left(x^{2}\right)$, and let $d$ be the discriminant of the polynomial $P(X)$. Let $L_{2}=K(\sqrt{a}, \sqrt{b})$. We denote by w the Hasse-Witt invariant of a quadratic form and by $(\cdot, \cdot)$ a Hilbert symbol. In [2], we saw that the embedding problem $2^{ \pm} S_{4} * Q_{8} \rightarrow S_{4} \times V_{4} \simeq \operatorname{Gal}(L \mid K)$ is solvable if and only if

$$
\mathrm{w}\left(Q_{E}\right)=( \pm 2, d) \cdot(a, b) \cdot(-1, a b) \text { in } H^{2}\left(G_{K},\{ \pm 1\}\right) .
$$

From now on, we assume that $K$ is a field of characteristic 3 , containing $\mathbb{F}_{9}$. We write $P(X)=X^{4}+s_{2} X^{2}-s_{3} X+s_{4}$. By computation of the trace form $Q_{E}$, we obtain

$$
\mathrm{w}\left(Q_{E}\right)=\left(d s_{2},\left(s_{2}^{2}-s_{4}\right) s_{4}\right) .
$$

The equivalent condition (2) for the solvability of the embedding problem (11) then turns into

$$
\left(d s_{2},\left(s_{2}^{2}-s_{4}\right) s_{4}\right)=(a, b),
$$

that is, the equality of two Hilbert symbols.

Now, taking into account that $K$ contains $\mathbb{F}_{9}$, the equality of Hilbert symbols (3) holds if and only if the two quadratic forms $\left\langle d s_{2}, m s_{4}, d m s_{2} s_{4}\right\rangle$ (where $m=s_{2}^{2}-s_{4}$ ) and $\langle a, b, a b\rangle$ are $K$-equivalent (see [5, 3.2]).

Remark 1 . We note that if $s_{2}=0$ or $s_{2}^{2}-s_{4}=0$, the embedding problem $2 S_{4} \rightarrow$ $S_{4} \simeq \operatorname{Gal}\left(L_{1} \mid K\right)$ is solvable. In this case, $L_{1}$ is embeddable in a $2 S_{4} * Q_{8}$-extension of $K$ if and only if there exists a $V_{4}$-extension $L_{2} \mid K$ disjoint with $L_{1}$ and such that the embedding problem $Q_{8} \rightarrow V_{4} \simeq \operatorname{Gal}\left(L_{2} \mid K\right)$ is solvable. Any $2 S_{4} * Q_{8}$-extension of $K$ containing $L_{1}$ is then obtained as $L(\sqrt{\alpha \beta})$, for $L=L_{1} \cdot L_{2}, L_{1}(\sqrt{\alpha})$ a solution to $2 S_{4} \rightarrow S_{4} \simeq \operatorname{Gal}\left(L_{1} \mid K\right)$ and $L_{2}(\sqrt{\beta})$ a solution to $Q_{8} \rightarrow V_{4} \simeq \operatorname{Gal}\left(L_{2} \mid K\right)$, with $L_{2}$ a $V_{4}$-extension of $K$ with the conditions above. The element $\alpha$ can be computed explicitly by the method obtained in [3] the element $\beta$ is given by the formula of Witt given in [7].

Theorem 1. Let $K$ be a field of characteristic 3 and containing $\mathbb{F}_{9}$. Let $P(X)=$ $X^{4}+s_{2} X^{2}-s_{3} X+s_{4} \in K[X]$ be a polynomial with discriminant $d$ and Galois group $S_{4}$, and let $L_{1}$ be its splitting field. Let $A \in \mathrm{GL}(3, K)$ be such that $D=$ $A^{t}\left\langle d s_{2}, m s_{4}, d m s_{2} s_{4}\right\rangle A$ is a diagonal matrix of the form $\langle a, b, a b\rangle$ and such that 
$L_{2}=K(\sqrt{a}, \sqrt{b})$ is a $V_{4}$-extension of $K$, disjoint from $L_{1}$. Let us choose the matrix $A$ such that $\operatorname{det} A=a b /\left(d m s_{2} s_{4}\right)$. Let $L$ be the compositum of $L_{1}$ and $L_{2}$. Let $M$ be the matrix with entries in $L$ given by

$$
M=\left(\begin{array}{cccc}
1 & 0 & 0 & 0 \\
0 & \frac{1}{\sqrt{a d}} & 0 & 0 \\
0 & 0 & \frac{1}{\sqrt{b d}} & 0 \\
0 & 0 & 0 & \frac{1}{\sqrt{a b d}}
\end{array}\right) \cdot\left(\begin{array}{cccc}
1 & x_{1} & x_{1}^{2} & x_{1}^{3} \\
1 & x_{2} & x_{2}^{2} & x_{2}^{3} \\
1 & x_{3} & x_{3}^{2} & x_{3}^{3} \\
1 & x_{4} & x_{4}^{2} & x_{4}^{3}
\end{array}\right)
$$

where $\left(x_{1}, x_{2}, x_{3}, x_{4}\right)$ are the four roots of the polynomial $P(X)$ in $L_{1}$. Let $B \in$ $\mathrm{GL}(4, K)$ be the matrix

$$
B=\left(\begin{array}{ll}
1 & 0 \\
0 & A
\end{array}\right)
$$

Let $P$ be the matrix in $\mathrm{GL}(4, K)$ given by

$$
P=\frac{1}{m}\left(\begin{array}{cccc}
m & 0 & -s_{2} d+s_{3}^{2} s_{2}^{4} & -s_{2}^{3} s_{3} d \\
0 & d m & \left(s_{2}^{2}+s_{4}\right) m s_{2} s_{3} & \left(s_{2}^{2}+s_{4}\right) d m \\
0 & 0 & d-s_{3}^{2} s_{2}^{3} & s_{2}^{2} s_{3} d \\
0 & 0 & s_{3} s_{2}^{2} m & s_{2} d m
\end{array}\right) .
$$

Let $\gamma$ be the element in $L^{*}$ given by

$$
\gamma=\operatorname{det}(M P B+I) \text {. }
$$

Then the general solution to the embedding problem $2^{-} S_{4} * Q_{8} \rightarrow S_{4} \times V_{4} \simeq$ $\operatorname{Gal}(L \mid K)$ is $L\left(\sqrt{r \gamma_{1}}\right)$ for $r \in K^{*}$ and

$$
\gamma_{1}=(1+\sqrt{a})^{2}(1+\sqrt{b})^{2}(1+\sqrt{d})^{2} \gamma .
$$

The general solution to the embedding problem $2^{+} S_{4} * Q_{8} \rightarrow S_{4} \times V_{4} \simeq \operatorname{Gal}(L \mid K)$ is $L\left(\sqrt{r \gamma_{2}}\right)$ for $r \in K^{*}$ and

$$
\gamma_{2}=\sqrt{d} \gamma_{1}
$$

Proof. We can check that the matrix $P$ satisfies $P^{t} T P=\left\langle 1, d s_{2}, m s_{4}, d m s_{2} s_{4}\right\rangle$, where $T$ is the matrix of the quadratic trace form $Q_{E}$ in the basis $\left(1, x_{1}, x_{1}^{2}, x_{1}^{3}\right)$ and apply [[2], theorems 1 and 2]. In particular, the choice of the sign of the determinant of the matrix $A$ assures that the element $\gamma$ is nonzero.

Remark 2. For each of the two extensions $L\left(\sqrt{\gamma_{i}}\right) \mid K, i=1,2$, with $\gamma_{1}, \gamma_{2}$ given by the theorem, we consider the discriminant $\Delta_{i}$ of the basis $\left\{x_{1}^{i_{1}} x_{2}^{i_{2}} x_{3}^{i_{3}} a^{\varepsilon_{1}} b^{\varepsilon_{2}} \gamma_{i}^{\varepsilon_{3}}\right.$ : $\left.0 \leq i_{1} \leq 3,0 \leq i_{2} \leq 2, i_{3}=0,1, \epsilon_{1}, \epsilon_{2}, \epsilon_{3}=0,1 / 2\right\}$, where $x_{1}, x_{2}, x_{3}$ are three distinct roots of the polynomial $P(X)$ realizing $S_{4}$. We have

$$
\Delta_{1}=(d a b)^{96}((1-a)(1-b)(1-d))^{24} N_{L \mid K}(\gamma) ; \Delta_{2}=d^{12} \Delta_{1} .
$$

Corollary 1. Let $K$ and $L_{1}$ be as in the theorem and assume that the extension $L_{2}=K(\sqrt{a}, \sqrt{b}) \mid K$, with $a=d s_{2}, b=m s_{4}$, has Galois group $V_{4}$ and is disjoint from $L_{1}$. Then the element $\gamma$ in the theorem corresponding to this field $L_{2}$ is given in terms of the coefficients $s_{2}, s_{3}, s_{4}$ of the polynomial $P(X)$ realising the extension $L_{1} \mid K$, of three distinct roots $x_{1}, x_{2}, x_{3}$ of this polynomial $P(X)$ and the square roots 
of the elements $a$ and $b$, by

$$
\begin{aligned}
& \gamma=m^{2} s_{2} s_{4} \sqrt{a} x_{1}^{3} x_{2}^{2}+2 m^{2} s_{2} s_{4} \sqrt{a} x_{1}^{3} x_{2} x_{3}+\left(2 m s_{2} d \sqrt{b}+\left(2 s_{3} s_{4} m\right.\right. \\
& \left.\left.+2 s_{3} m^{2}\right) \sqrt{a b}\right) x_{1}^{3} x_{2}+\left(s_{4}^{2} m^{2} \sqrt{a}+\left(2 s_{4} m^{3} s_{3}+d s_{3} s_{4}+s_{4}^{2} s_{2} s_{3}^{3}+m^{2} s_{3}^{3} s_{2}\right.\right. \\
& \left.+d s_{3} m+2 s_{3} s_{4}^{2} m^{2}+2 m s_{4} s_{2} s_{3}^{3}\right) \sqrt{b}+\left(2 s_{4} m^{2}+d+m s_{2} s_{3}^{2}\right. \\
& \left.\left.+s_{4} s_{2} s_{3}^{2}\right) \sqrt{a b}\right) x_{1}^{3}+\left(d s_{2}+2 s_{4} m s_{3}^{2}+s_{3}^{2} m^{2}+s_{3}^{2} s_{4}^{2}\right) \sqrt{a} x_{1}^{2} x_{2}^{2} x_{3}+\left(\left(2 s_{3} m^{2}\right.\right. \\
& \left.\left.+2 s_{3} s_{4} m\right) \sqrt{a b}\right) x_{1}^{2} x_{2}^{2}+\left(m s_{2} d \sqrt{b}+\left(2 s_{3} m^{2}+2 s_{3} s_{4} m\right) \sqrt{a b}\right) x_{1}^{2} x_{2} x_{3} \\
& +\left(\left(2 d m+2 s_{4} m^{3}+2 m^{2} s_{3}^{2} s_{2}+2 s_{4}^{2} m^{2}+2 m s_{4} s_{2} s_{3}^{2}\right) \sqrt{a}+\left(2 d s_{3} m\right.\right. \\
& \left.\left.+2 d s_{3} s_{4}\right) \sqrt{b}\right) x_{1}^{2} x_{2}+\left(\left(2 s_{2} s_{4}^{2} s_{3}^{2}+2 m s_{4} s_{2} s_{3}^{2}+2 s_{4}^{2} m^{2}+2 d s_{4}\right) \sqrt{a}\right. \\
& +\left(s_{3} s_{4}^{2} m^{2}+s_{4} m^{3} s_{3}+d s_{3} s_{4}+2 m^{2} s_{3}^{3} s_{2}+2 s_{4}^{2} s_{2} s_{3}^{3}+d s_{3} m+m s_{4} s_{2} s_{3}^{3}\right) \sqrt{b} \\
& \left.+\left(s_{4} m^{2}+m s_{2} s_{3}^{2}+s_{4} s_{2} s_{3}^{2}\right) \sqrt{a b}\right) x_{1}^{2} x_{3}+\left(2 m^{2} s_{2} s_{4} s_{3}+s_{4} m s_{3}^{3}+2 s_{3}^{3} m^{2}\right. \\
& \left.+2 d s_{2} s_{3}+2 s_{4}^{2} s_{3}^{3}\right) \sqrt{a} x_{1}^{2}+\left(m s_{2} d \sqrt{b}+\left(2 s_{3} m^{2}+2 s_{3} s_{4} m\right) \sqrt{a b}\right) x_{1} x_{2}^{2} x_{3} \\
& +\left(\left(2 m s_{4} s_{2} s_{3}^{2}+2 s_{4}^{2} m^{2}+2 m^{2} s_{3}^{2} s_{2}+2 d m\right) \sqrt{a}+\left(2 d s_{3} m+s_{3} s_{4}^{2} m^{2}\right.\right. \\
& \left.+2 s_{4}^{2} s_{2} s_{3}^{3}+2 m^{2} s_{3}^{3} s_{2}+2 d s_{3} s_{4}+m s_{4} s_{2} s_{3}^{3}+s_{4} m^{3} s_{3}\right) \sqrt{b} \\
& \left.+\left(s_{4} s_{2} s_{3}^{2}+m s_{2} s_{3}^{2}+s_{4} m^{2}\right) \sqrt{a b}\right) x_{1} x_{2}^{2} \\
& +\left(\left(m^{2} s_{3}^{2} s_{2}+s_{4}^{2} m^{2}+d m+m s_{4} s_{2} s_{3}^{2}\right) \sqrt{a}+\left(2 m s_{4} s_{2} s_{3}^{3}+m^{2} s_{3}^{3} s_{2}\right.\right. \\
& \left.\left.+2 s_{3} s_{4}^{2} m^{2}+s_{4}^{2} s_{2} s_{3}^{3}+2 s_{4} m^{3} s_{3}\right) \sqrt{b}+\left(2 d+2 s_{4} m^{2}\right) \sqrt{a b}\right) x_{1} x_{2} x_{3} \\
& +\left(\left(2 s_{4}^{2} s_{3}^{3}+2 s_{3}^{3} m^{2}+2 d s_{2} s_{3}+s_{4} m s_{3}^{3}\right) \sqrt{a}+\left(2 d s_{4} m+2 d m^{2}\right) \sqrt{b}\right) x_{1} x_{2} \\
& +\left(\left(s_{3}^{3} m^{2}+m^{2} s_{2} s_{4} s_{3}+2 s_{4} m s_{3}^{3}+d s_{2} s_{3}+s_{4}^{2} s_{3}^{3}\right) \sqrt{a}\right) x_{1} x_{3} \\
& +\left(\left(2 m^{2} s_{2} s_{4}^{2}\right) \sqrt{a}+\left(2 m s_{2} d s_{3}+2 m^{3} s_{3} s_{2} s_{4}+2 m^{2} s_{2} s_{4}^{2} s_{3}+m^{3} s_{3}^{3}\right.\right. \\
& \left.\left.+s_{4}^{3} s_{3}^{3}\right) \sqrt{b}+\left(s_{4}^{2} s_{3}^{2}+d s_{2}+2 s_{3}^{2} m^{2}+2 m^{2} s_{2} s_{4}\right) \sqrt{a b}\right) x_{1}+\left(\left(2 d s_{4}\right.\right. \\
& \left.+2 m s_{4} s_{2} s_{3}^{2}+2 s_{2} s_{4}^{2} s_{3}^{2}\right) \sqrt{a}+\left(2 d s_{3} m+2 d s_{3} s_{4}\right) \sqrt{b}+\left(2 m s_{2} s_{3}^{2}+d\right. \\
& \left.\left.+2 s_{4} s_{2} s_{3}^{2}\right) \sqrt{a b}\right) x_{2}^{2} x_{3}+\left(2 d s_{2} s_{3}+2 s_{3}^{3} m^{2}+2 s_{4}^{2} s_{3}^{3}+s_{4} m s_{3}^{3}\right) \sqrt{a} x_{2}^{2} \\
& +\left(2 s_{4} m s_{3}^{3}+s_{3}^{3} m^{2}+d s_{2} s_{3}+s_{4}^{2} s_{3}^{3}\right) \sqrt{a} x_{2} x_{3}+\left(m^{2} s_{2} s_{4}^{2} \sqrt{a}+\left(d s_{4} s_{2} s_{3}\right.\right. \\
& \left.+m^{3} s_{3}^{3}+2 m^{3} s_{3} s_{2} s_{4}+2 m^{2} s_{2} s_{4}^{2} s_{3}+s_{4}^{3} s_{3}^{3}\right) \sqrt{b}+\left(2 m^{2} s_{2} s_{4}+2 d s_{2}\right. \\
& \left.\left.+s_{3}^{2} m^{2}+s_{4} m s_{3}^{2}\right) \sqrt{a b}\right) x_{2}+\left(\left(s_{3}^{2} s_{4}^{3}+2 s_{3}^{2} s_{4}^{2} m+m^{2} s_{2} s_{4}^{2}+s_{4} m^{2} s_{3}^{2}\right.\right. \\
& \left.+s_{2} s_{4} d\right) \sqrt{a}+\left(2 s_{4}^{3} s_{3}^{3}+2 m^{3} s_{3}^{3}+m s_{2} d s_{3}+m^{3} s_{3} s_{2} s_{4}+m^{2} s_{2} s_{4}^{2} s_{3}\right) \sqrt{b} \\
& \left.+\left(m^{2} s_{2} s_{4}+d s_{2}+2 s_{4} m s_{3}^{2}+2 s_{3}^{2} m^{2}\right) \sqrt{a b}\right) x_{3}+m^{2} s_{2} s_{4} d \\
& +\left(s_{2} s_{4}^{2} s_{3}^{3}+d s_{4} s_{3}+m s_{4} s_{2} s_{3}^{3}\right) \sqrt{a}+\left(d s_{3}^{2} s_{4}+d s_{3}^{2} m+m s_{4} s_{2} d\right) \sqrt{b} \\
& +\left(2 d s_{3}+m s_{2} s_{3}^{3}+s_{2} s_{3}^{3} s_{4}\right) \sqrt{a b} \text {. }
\end{aligned}
$$

Proof. We apply the theorem with the matrix $B$ equal to the identity matrix. The computation of the corresponding determinant is simplified by applying the Cramer identities. The stated expression for the element $\gamma$ is obtained by using the symmetric functions in the four roots $x_{1}, x_{2}, x_{3}, x_{4}$ of the polynomial $P(X)$. The computations are carried out with Maple. 
Example 1. The polynomial $P(X)=X^{4}+\left(Y_{1}+Y_{2}\right) X^{2}+Y_{2} X+\left(Y_{1}+Y_{3}\right)$ has Galois group $S_{4}$ over the field $K=\overline{\mathbb{F}}_{3}\left(\left(Y_{1}, Y_{2}, Y_{3}\right)\right)$. Let $L_{1}$ be the splitting field of $P(X)$. We write $s_{2}=Y_{1}+Y_{2}, s_{3}=-Y_{2}, s_{4}=Y_{1}+Y_{3}$ and consider the elements $a=d s_{2}, b=\left(s_{2}^{2}-s_{4}\right) s_{4}$. To assure that the extension $L_{2}=K(\sqrt{a}, \sqrt{b}) \mid K$ is a $V_{4}$-extension disjoint from $L_{1}$ we check that the elements $a, b, a b, s_{2}, d b, s_{2} b$ are not squares in $K$. To this end we use the following lemma, which is a direct consequence of the Weierstrass preparation theorem (see e.g. [8]).

Lemma 1. Let $F \in R=\overline{\mathbb{F}}_{3}\left[\left[Y_{1}, Y_{2}, Y_{3}\right]\right]$ be regular and polynomial in $Y_{i}$ for some $i=1,2,3$. If the discriminant $\Delta\left(F, Y_{i}\right)$ is nonzero, then $F$ is not a square in $K$.

Let $L$ be the composition field of $L_{1}$ and $L_{2}$. By applying the corollary, we obtain extensions $L\left(\sqrt{\gamma_{1}}\right) \mid K$ and $L\left(\sqrt{\gamma_{2}}\right) \mid K$ with Galois groups $2^{-} S_{4} * Q_{8}$ and $2^{+} S_{4} * Q_{8}$, respectively, with an explicit expression for the elements $\gamma_{1}$ and $\gamma_{2}$.

\section{REFERENCES}

[1] S. S. Abhyankar, Resolution of singularities and modular Galois theory, Bull. Amer. Math. Soc. 38 (2001), 131-169. MR 2002a:14013

[2] T. Crespo, $2 S_{4} * Q_{8}$-extensions, Algebra, Arithmetic and Geometry with Applications, Papers from Shreeram S. Abhyankar's 70th Birthday Conference, C. Christensen, G. Sundaram, and A. Sathaye (eds.), Springer-Verlag, New York, 2003, to appear.

[3] T. Crespo, Explicit construction of $2 S_{n}$ Galois extensions, J. Algebra 129 (1990), 312-319. MR 91d:11135

[4] D. Harbater, M. van der Put and R. Guralnick, Valued fields and covers in characteristic p, Fields Institute Communications 32 (2002), 175-204.

[5] T. Y. Lam, The algebraic theory of quadratic forms, Benjamin/Cummings Publ. Co., Reading, MA, 1973. MR 53:277

[6] T. R. Lefcourt, Galois groups and complete domains, Israel J. Math 114 (1999), 323-346. MR 2000k:13006

[7] E. Witt, Konstruktion von galoischen Körpern der Charakteristik p zu vorgegebener Gruppe der Ordnung $p^{f}$, J. Crelle 174 (1936), 237-245.

[8] O. Zariski and P. Samuel, Commutative Algebra II, Graduate Texts in Math. 29, SpringerVerlag, New York, 1976. MR 52:10706

Departament d’Àlgebra i Geometria, Universitat de Barcelona, Gran Via de les Corts Catalanes 585, 08007 Barcelona, Spain

E-mail address: crespo@cerber.mat.ub.es 